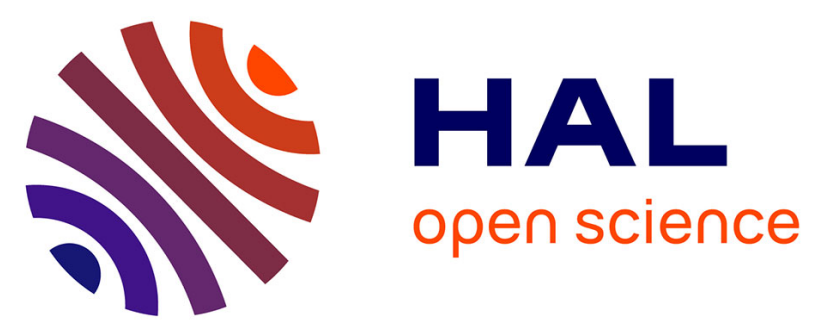

\title{
Adapting numerical representations of lung contours using Case-Based Reasoning and Artificial Neural Networks
}

Julien Henriet, Pierre-Emmanuel Leni, Rémy Laurent, Ana Roxin, Brigitte Chebel-Morello, Michel Salomon, Jad Farah, David Broggio, Didier Franck, Libor Makovicka

\section{To cite this version:}

Julien Henriet, Pierre-Emmanuel Leni, Rémy Laurent, Ana Roxin, Brigitte Chebel-Morello, et al.. Adapting numerical representations of lung contours using Case-Based Reasoning and Artificial Neural Networks. ICCBR 2012, 20th International Conference on Case-Based Reasoning, Sep 2012, Lyon, France. pp.137 - 151, 10.1007/978-3-642-32986-9_12 . hal-00935864

\section{HAL Id: hal-00935864 https://hal.science/hal-00935864}

Submitted on 24 Jan 2014

HAL is a multi-disciplinary open access archive for the deposit and dissemination of scientific research documents, whether they are published or not. The documents may come from teaching and research institutions in France or abroad, or from public or private research centers.
L'archive ouverte pluridisciplinaire HAL, est destinée au dépôt et à la diffusion de documents scientifiques de niveau recherche, publiés ou non, émanant des établissements d'enseignement et de recherche français ou étrangers, des laboratoires publics ou privés. 


\title{
Adapting numerical representations of lung contours us- ing Case-Based Reasoning and Artificial Neural Net- works
}

\author{
J. Henriet ${ }^{1, *}$, P-E. Leni ${ }^{1}$, R. Laurent ${ }^{1}$, A. Roxin ${ }^{1}$, B. Chebel-Morello ${ }^{2}$, M. Salo- \\ mon $^{2}$, J. Farah ${ }^{3}$, D. Broggio ${ }^{3}$, D. Franck ${ }^{3}$, L. Makovicka ${ }^{1}$ \\ ${ }^{1}$ CE UMR 6249 CNRS, Université de Franche-Comté, France \\ $\{$ henriet, leni, laurent, roxin, makovicka\}@univ-fcomte.fr \\ ${ }^{2}$ FEMTO-ST UMR 6174 CNRS, Université de Franche-Comté, France \\ \{morello, salomon \} @univ-fcomte.fr \\ ${ }^{3}$ French Institute of Radiological Protection and Nuclear Safety, France \\ \{farah, broggio, franck\} @irsn.fr
}

\begin{abstract}
In case of a radiological emergency situation involving accidental human exposure, a dosimetry evaluation must be established as soon as possible. In most cases, this evaluation is based on numerical representations and models of subjects. Unfortunately, personalised and realistic human representations are often unavailable for the exposed subjects. However, accuracy of treatment depends on the similarity of the phantom to the subject. The EquiVox platform (Research of Equivalent Voxel phantom) developed in this study uses Case-Based Reasoning principles to retrieve and adapt, from among a set of existing phantoms, the one to represent the subject. This paper introduces the EquiVox platform and Artificial Neural Networks developed to interpolate the subject's 3D lung contours. The results obtained for the choice and construction of the contours are presented and discussed.
\end{abstract}

Keuwords: Adaptation, Interpolation, Case-Based Reasoning, Artificial Neural Network, 3D personalised phantoms.

\section{Introduction}

In case of accidental exposure to radiation, a dosimetry evaluation must be established for each potential victim (subject) as soon as possible. In most cases, this evaluation is based on available 3D voxel Phantoms, numerical models created from medical images to represent the imaged subject with maximum realism. Examples of voxel phantoms for dosimetric assessment following internal contamination or external exposure can be found [1], [2]. However, even when medical images are available, the subject's specific phantom is not always accessible since its construction is delicate and time consuming, and in emergency cases such time and effort are unaffordable. Moreover, medical images are avoided so as to prevent any additional exposure to radiation. Thus, existing models are used even if their characteristics differ from the subject's biometrical data. Dosimetry assessment accuracy and the resulting decontaminating medical action is nevertheless highly dependent on the similarity 
between phantom and subject. Hence, the actual work aims at assisting the physician in choosing and customizing the most similar phantom from the existing and available ones.

Case-Based Reasoning (CBR) is a problem solving method that uses similar solutions from similar past problems in order to solve new problems [3]. The EquiVox platform uses the CBR-approach to find the most similar phantom(s) within any set of phantoms and then attempts to adapt them to the characteristics of the target case (the subject). EquiVox adaptation tool uses Artificial Neural Networks [4] to adapt the stored phantoms to the subject.

A large number of phantoms can be found in literature [5], [6] and radiation protection is also divided into numerous sub-domains. Indeed, some phantoms are commonly used by experts for external radiotherapy, and others are used by other physicians for evaluation of internal doses received. In fact, each expert has his own collection of 10 to 20 phantoms. When physician's usual phantoms are all too distant from the subject, the expert must create a new one. Indeed, using iterative 3D dilations and contractions, physicians modify the contours of the $3 \mathrm{D}$ organs of their phantoms until they correspond to those of the subject.

Then, they put them together and obtain the final phantom on which the computations will be based [6]. Thus, the adaptation rules are guided by their experience and knowledge. The main challenge of EquiVox is to reproduce the same transformation process automatically, without human intervention. Another requirement of EquiVox is to be able to use any set of phantoms and to help the physician to capitalise on them. We also hope that such a platform will be used to automatically create a wellfitting phantom for each subject in order to increase the accuracy of dose calculations. At this step of the implementation, we relied on phantoms usually used by a team of experts for pulmonary anthroporadiametry which consists of evaluating the internal dose inhaled.

\section{The EquiVox application}

Figure 1 presents the technologies that were used and the data flows over the EquiVox architecture. All the phantoms are stored in Rhino3D files [7]. Their characteristics are stored in a database (data flow \#0 in Figure 1), the lung contours are extracted (data flow \#1) and then transmitted to the ANN training module (data flow \#2) which creates the ANN (data flow \#3).

When a new phantom is required, the target case description is transmitted to the retrieval module (data flow \#4) which determines the similitude and confidence indices taking into account the source case (data flow \#5).

If required by the experts, the lung adaptation module sends the characteristics of the source cases (data flow \#6) to the ANN interpolation module (data flow \#7) which loads the trained ANN (data flow \#8) and the coordinates of the contour of the lungs 
in question (data flow \#9) in order to create interpolated contours suited to the target case (data flow \#10).

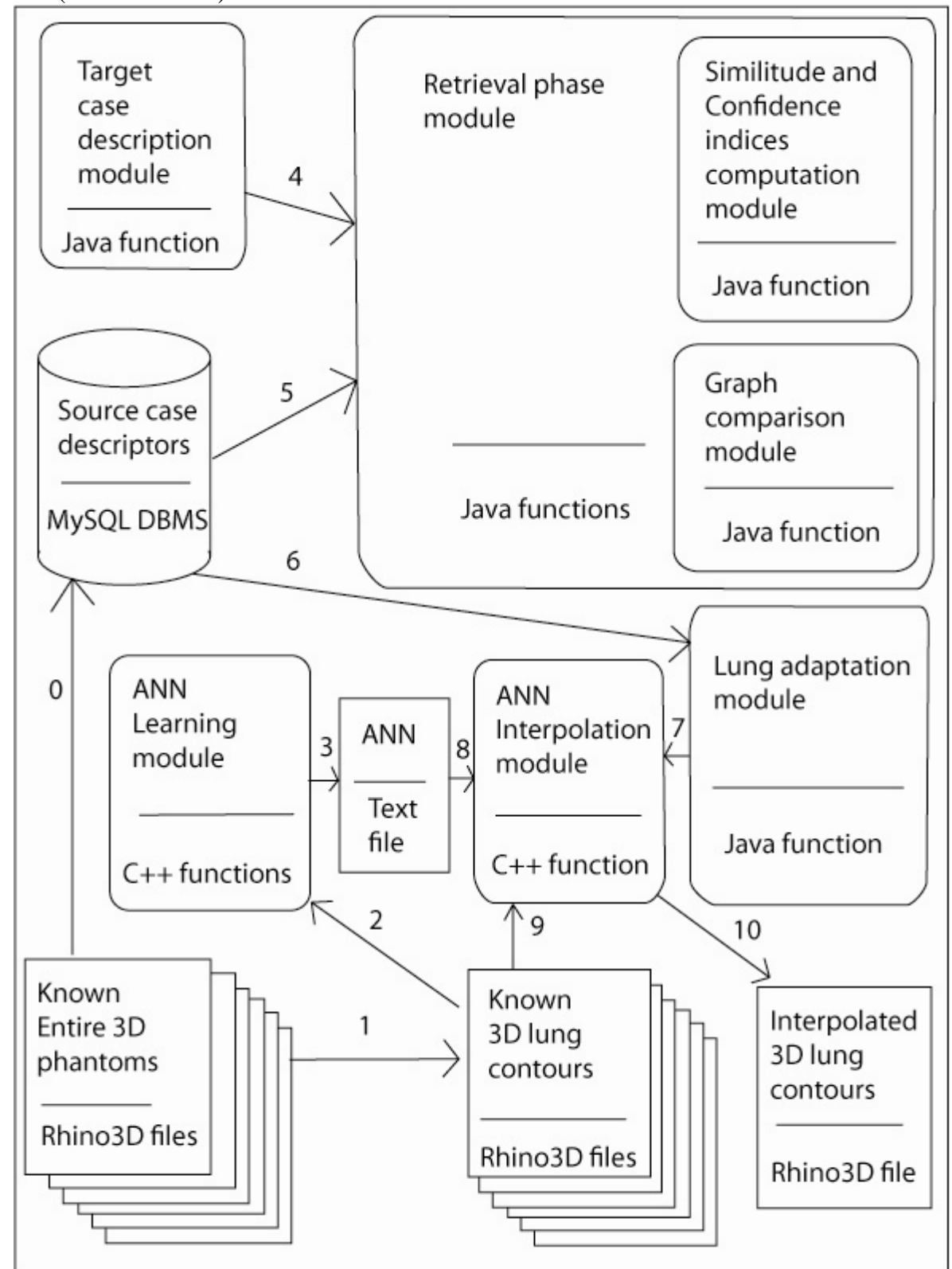

Fig. 1. Data flows over the EquiVox architecture.

It is to notice that the adaptation module of EquiVox is not complete yet. Since lungs are the first organs that are designed by experts, we focused on their adaptation while the EquiVox retrieval phase is able to compare the entire phantoms. Thus, the 
adaptation module of EquiVox deals with the Lung Contours in 3 Dimensions (3DLC). Other studies have been begun to focus on the adaptation of the other organs.

\subsection{Case modelling}

When radiation overexposure occurs, a dosimetric report must be established for all subjects. For each subject, the experts' first task is to choose the most accurate 3D phantom considering the information known about the subject. Each phantom has its own characteristics and is chosen by comparing the subject's available measurements and information to his/her characteristics. The phantom is thus chosen by analogy.

As explained, the experts choose the phantom according to the characteristics of the subject. We exhausted the list of useful characteristics furnished by the physicians of the French Institute of Radiation and Protection (IRSN).

Thus, in EquiVox, a problem is described as a set of $r$ descriptors $\left\{d_{1}, \ldots, d_{r}\right\}$.

Each expert has his own set of $n$ phantoms $\left\{P_{i}, \ldots, P_{n}\right\}$.

Each $P_{i}$ is the solution part of a case and represents the contours of $m$ organs: $P_{i}=\left\{P_{i}^{1}, \ldots, P_{i}^{m}\right\}$.

Each organ $O$ is a set of $q$ points joined by a Delauney mesh [8]: $P_{i}^{O}=$ $\left\{C_{1}^{i, o}, \ldots, C_{q}^{i, o}\right\}$ where $C_{j}^{i, o}$ denotes the $3 \mathrm{D}$ coordinates of point $j$ of organ $O$ of phantom $P_{i} . O \in$ \{lungs, heart, liver, sternum, ribs, scapulae, spine, breast, skin, oesophagus\}.

Finally, a case $i$ is equal to $\left\{\left\{d_{1}^{i}, \ldots, d_{r}^{i}\right\}, P_{i}\right\}$. We will note $t$ as target case.

\subsection{Retrieval phase}

The purpose of this phase is to sort the phantoms of the EquiVox case-base according to information concerning the subject, even if incomplete. Hence, the number of known descriptors influences the level of confidence in the proposed EquiVox ranking. Thus, along with the similarity index $\left(S_{i}\right)$, a confidence index $(C)$ is assessed to associate the probable error with the retrieved solution.

In addition, some descriptors may be very important for some types of calculations while others may be totally neglected. Since the purpose of EquiVox is to retrieve and adapt phantoms, whatever their use, our platform must take into account the importance of each descriptor. Thus, the descriptors were weighted, taking into account their importance and influence. As presented in Equations (1) and (2), these weights $\left\{\omega_{1}, \ldots, \omega_{r}\right\}$ are quantitative values associated to each descriptor, amplifying or reducing the differences between $t$ and $i$. They thus stress on the relative influence that one measure represents in comparison to the others.

In fact, when a new problem occurs, some of the subject's characteristics may be unavailable. Thus, a Boolean value $\delta_{k}$ is associated to each $d_{k}$. $\delta_{k}$ is equal to 0 if the value of $d_{k}$ is unknown, and to 1 otherwise. 
Hence, a classical algorithm for similarity calculation was used, namely the K-nn Algorithm that enables a weight to be applied to the descriptor values.

The $S_{i}$ value is equivalent to the sum of the distances between the descriptors of $i$ and $t$, each weighted accordingly. It is given by the following equation:

$$
S_{i}=\frac{\sum_{k=1}^{r} \delta_{k} \cdot \omega_{k} \cdot\left[\frac{\Delta_{k}-\left|d_{k}^{i}-d_{k}^{t}\right|}{\Delta_{k}}\right]}{\sum_{k=1}^{r} \delta_{k} \cdot \omega_{k}}
$$

where $\Delta_{k}$ is the difference between the maximum and the minimum known values that the descriptor $d_{k}$ can take. The $S_{i}$ value is always between 0 and 1 . The greater the similarity of $i$ to $t$, the closer the $S_{i}$ value is to 1 .

Since $S_{i}$ only takes into account the known values of $t$, the confidence index $C$ must be taken into account to define the calculation uncertainty. The more values we know, the higher the confidence index. Indeed, if the subject's age is the only known criteria, the similarity value calculated is totally insignificant. So $C$ takes into account the number of known values according to the following formula:

$$
C=\frac{\sum_{k=1}^{r} \delta_{k} \cdot \omega_{k}}{\sum_{k=1}^{r} \omega_{k}}
$$

\subsection{Adaptation of $3 D$ lung contours}

Once a matching case is retrieved, the expert can decide either to use the phantom of the most similar source cases, or require the EquiVox platform to generate a new phantom, adapting the source cases to the target one. Indeed, if some available phantom measurements are too different from those of the subject, the expert may decide to adapt one of them or even to create a new phantom which may be reused for other problems later. Thus, when the expert requires the generation of a new phantom, the contours of the $m$ organs are expected.

Actually, the first organs experts create in such a personalised process are the lungs. The positions and volumes of the other organs are deduced from the lungs. Thus, we first considered the adaptation of 3D Lung Contours (3DLC).

Solution space modelling for 3D lung contours. As presented in the case modelling part, the lung contours of phantom $P_{i}$ are defined in 3D by a set of $q$ points joined by a Delaunay mesh: $P_{i}^{\text {lung }}=\left\{C_{1}^{i, \text { lung }}, \ldots, C_{q}^{i, \text { lung }}\right\}$ where $C_{j}^{i, \text { lung }}$ denotes the 3D coordinates of point $j: C_{j}^{i, l u n g}=\left\{x_{j}^{i, l \text { lung }}, y_{j}^{i, \text { lung }}, z_{j}^{i, \text { lung }}\right\}$.

For all the phantoms, the same number of points defines the $3 \mathrm{D}$ contours of the lungs: $q=26723$. The points have been plotted in the same order and in the same Cartesian coordinate system. Thus, the task of the lung contour-adaptation phase of EquiVox consists of interpolating the 3D coordinates of the points of $t$ in the same 
order and in the same Cartesian coordinate system. A Delaunay mesh can then be applied so as to create the contours of the lungs of $t$.

Adaptation rules. Not all the descriptors that identify the phantoms contained in EquiVox are useful for the adaptation of the lungs. Precisely, it has been proven by I. Clairand et al. that the height of a person prevails for the geometry and volume of its lungs [9].

Thus, when experts decide to create the lung contours of a subject, they choose the lung contours of the stored phantom whose height is the closest without taking into account any other characteristic. The adaptations are usually done manually, applying mathematical transformations (2D and 3D contractions and dilations [6]). These transformations are carried out through 3D modelling tools (such as Rhinoceros [7]).

In addition, these transformations are only driven by experience, trials and errors, and may take many hours or more. The delay also increases with the number of subjects whereas the problem resolution delay may be limited. Indeed, in the case of massive irradiation for example, when a disaster such as a nuclear explosion occurs, dosimetric reports are required for hundreds of people of different sizes.

In fact, the creation of new lung contours requires a fast data-driven method, and since there is no physical law to governing its design, the expert is not able to explicit a rule for the transformation of the lung contours.

Method. Since the mesh and the number of points are not variable, the adaptation must be carried out on the point coordinates of the lung contours, point by point. Since no formal equation exists, we must discover through a learning method the rules that transform the coordinates of the points on one lung contour into other coordinates.

Consequently, data-driven methods using inductive reasoning are the most suitable approaches; ANN and Fuzzy-ANN respond to these requirements. We chose ANN as the tool for this step, assuming this could serve as the basis for further work with Fuzzy-ANN if the first results were not convincing. We explored the possibility of using perceptrons with one hidden layer trained with a backpropagation-based method.

To interpolate the 3D lung contours, the height is required. Actually, this is one of the descriptors of the EquiVox target and source cases. Let us note $h_{i}$ the descriptor corresponding to the height of the case $i$ and $h_{t}$, the height of the target case $t$. Each $C_{j}^{t, \text { lung }}$ of $t$ is interpolated from $C_{j}^{i, l u n g}, h_{i}$ and $\Delta_{h}=h_{i}-h_{t}$ where $i$ is the case for which $\left|\Delta_{h}\right|$ is the smallest. 


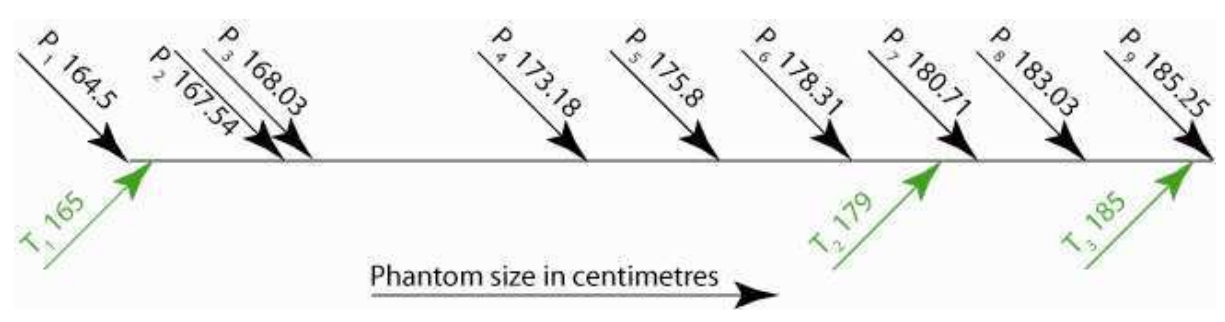

Fig. 2. Phantom heights of the available 3D lung contours.

In Figure 2, the 9 heights of the 3DLC $P_{1}$ to $P_{9}$ used for the training are reported on the axis. Other 3DLC were also drawn for the test set. The 3 heights of these 3DLC $T_{1}, T_{2}$ and $T_{3}$ are also reported on the same axis. All the thorax organs are represented in $P_{1}$ to $P_{9}$ whereas only the lungs were drawn in $T_{1}, T_{2}$ and $T_{3}$.

Training ends when the difference between the expected and the obtained values is minimised. W. Hsieh [10] distinguished four algorithms based on the backpropagation method:

- The BFGS method (Broyden-Fletcher-Goldfarb-Shanno) is a quasiNewton method, which approximates the value of the Hessian matrix of the second derivatives of the function to be minimised;

- The L-BFGS method (Limited memory - BFGS) is an adaptation of the BFGS method which optimises the computational resources to use. Both of these methods must be coupled with a Wolfe linear search in order to determine an optimal step size between two iterations;

- The Rprop (Resistant backpropagation) method proposes a first order algorithm but its complexity increases linearly with network topology;

- The iRpropPlus method is one of the fastest and also one of the most accurate algorithms. This evolution of the Rprop method allows cancelling some synaptic weight updates in the neural network if a negative effect is observed.

Table 1. ANN configuration with which the best preliminary results.

\begin{tabular}{ccc}
\hline Phantom height $[\mathrm{cm}]$ & Required precision & Best Learning method \\
\hline 178.31 & $10^{-6}$ & BFGS \\
180.71 & $10^{-6}$ & BFGS \\
183.03 & $10^{-6}$ & BFGS \\
\hline
\end{tabular}

These methods were previously implemented and tested in the EquiVox adaptation phase of 3DLC. Different required precisions were also tested. The coordinates of 10 points were randomly extracted from the 3DLC of $P_{1}$ to $P_{9}$ and a cross validation was performed. Table 1 shows the algorithm that gave the best interpolations is the one with BFGS as backpropagation method and a precision equals to $10^{-6}$. 
Then, the chosen ANN configuration was compared to a polynomial (Newton, of degree 2) and a Spline interpolation method. The Newton interpolation function proposed by J. Ponce and R. Brette in [11] and the Spline one proposed by Scilab [12]. were implemented with Scilab 5.3.2.

For each method, a cross-validation for the same 10 points was undertaken using the same 3DLC of $P_{1}$ to $P_{9}$. Figure 3 presents the mean distances between interpolated and expected coordinates. This figure shows that the polynomial interpolation produced the greatest errors among the three tested interpolations. A factor nearly equal to 10 can be observed between the polynomial interpolation and that of the Spline or the ANN. The Spline and the ANN interpolations gave closer errors. Nevertheless, for all the tested cases, the ANN interpolation errors were inferior to the Spline ones 6 times and equal only once. These results prove the superiority of the ANN interpolations over the other methods since the ANN interpolation gave a more accurate result in all the tested cases.

Actually, during the training phase of ANN, learning sets are generally divided in two parts: some of the elements are used to learn while others are used to validate. During this step, the number of neurons of the hidden layers is also determined. Since the number of 3DLC of our learning set is limited, we wanted to study the impact of some 3DLC in the learning. Thus, we defined two main configurations and four possibilities for each.

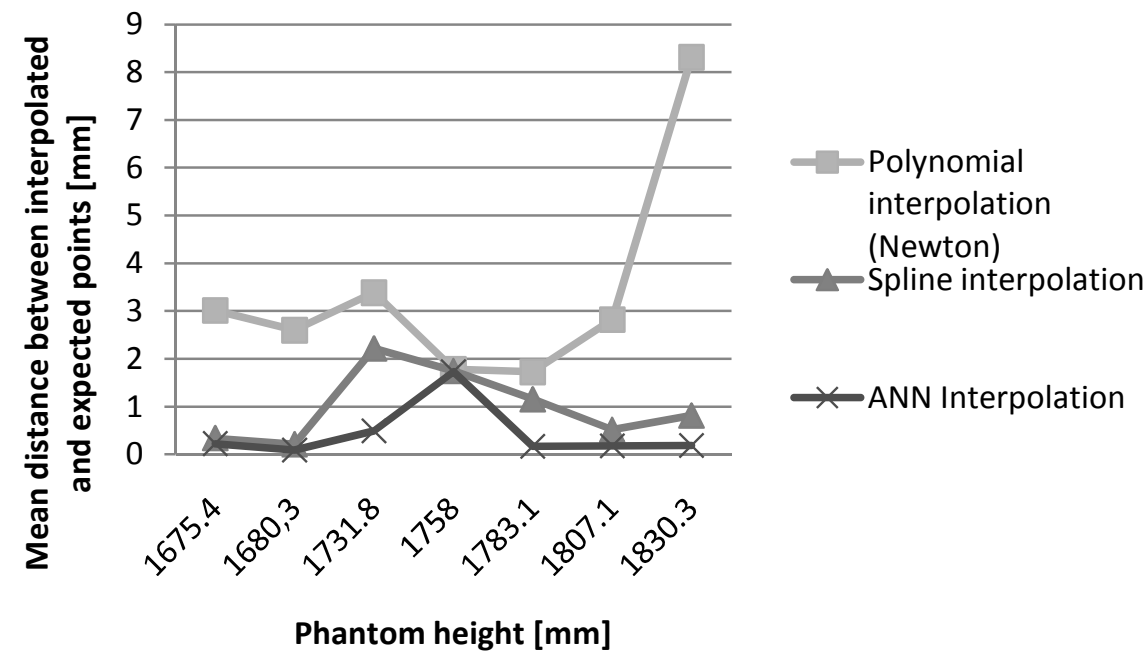

Fig. 3. Mean distances obtained between interpolated and expected coordinates for 10 points and 3 interpolation algorithms.

Table 2. Learning, validation and test sets tested.

\begin{tabular}{cccc}
\hline & Learning set & Validation set & Test set \\
\hline Possibility \#1 & $\left\{P_{1}, P_{3}, P_{4}, P_{5}, P_{6}, P_{7}, P_{8}, P_{9}\right\}$ & $\left\{P_{2}\right\}$ & $\left\{T_{1}, T_{2}, T_{3}\right\}$
\end{tabular}




\begin{tabular}{llll} 
Possibility \#2 & $\left\{P_{1}, P_{2}, P_{4}, P_{5}, P_{6}, P_{7}, P_{8}, P_{9}\right\}$ & $\left\{P_{3}\right\}$ & $\left\{T_{1}, T_{2}, T_{3}\right\}$ \\
Possibility \#3 & $\left\{P_{1}, P_{2}, P_{3}, P_{4}, P_{5}, P_{7}, P_{8}, P_{9}\right\}$ & $\left\{P_{6}\right\}$ & $\left\{T_{1}, T_{2}, T_{3}\right\}$ \\
Possibility \#4 & $\left\{P_{1}, P_{2}, P_{3}, P_{4}, P_{5}, P_{6}, P_{8}, P_{9}\right\}$ & $\left\{P_{7}\right\}$ & $\left\{T_{1}, T_{2}, T_{3}\right\}$ \\
\hline
\end{tabular}

For the first configuration the constraint over the ANN input $h_{i}-h_{t}=\Delta_{h}>0$ was added and for the second one $\Delta_{h}<0$ was required. Then for each configuration, we explored the possibility to extract one particular phantom of the learning set and to include it in the validation set. For each possibility, the test set was always the same: $\left\{T_{1}, T_{2}, T_{3}\right\}$ Table 2 shows the different possibilities that were tested.

\section{Results}

The Equivox platform has been implemented and tested on a Personal Computer equipped with an Intel Core i3 CPU, $2.53 \mathrm{GHz}$, and $4 \mathrm{GiB}$ RAM. The source case descriptors are stored in a mySQL database management system (DBMS). Two programming languages were used: Java and C. The retrieval phase, the GUIs, and the storage phase modules developed by our team in Java call C++ programs also developped by our team for the adaptation phase. The phantoms were drawn using Rhino3D.

The ANN learning was performed in $\mathrm{C}++$ on the supercomputer facilities of the Mésocentre de calcul de Franche-Comté, that contains 74 nodes based on Intel processors ( 4 or 6 cores) and 12 to 96 GB of ram. Each learning phase is monothread, so several learning phases could be simultaneously executed on one node.

\subsection{EquiVox case base}

The EquiVox case-base used for the tests contained 24 whole 3D phantoms with $3 \mathrm{D}$ organ contours and characteristics. These phantoms were manually designed from the ICRP standard female phantom [5] for pulmonary anthroporadiametry computations by the team of internal dose evaluation of IRSN [6]. These 3D phantoms were developed to cover as well as possible the diversity in the female population: thoracic phantoms of cup sizes ranging from A to F and chest girth from 85 to 120 (European Standard Clothing Units) [13].

These phantoms were developed for in vivo lung counting optimisation where volume and weight precisions are available for the following structures: lungs, heart, liver, sternum, ribs, scapulae, spine, breasts, skin, and oesophagus. The following external measurements are also available: age, sex, height, weight, cup size, and chest girth (chest and under-bust circumferences). Thus, all these female phantoms and characteristics formed the 24 source cases of the tested EquiVox case-base.

The experts determined a list of 14 descriptors having varying degrees of influence in the choice of phantom for this type of calculation. These descriptors are age, height, weight, sex, wether the subject smokes or not, thorax volume, lung volume, extrathoracic thickness, fat-muscle proportion, under-bust circumference, wrist di- 
ameter, chest circumference, heart volume, and the subject's origin (target case) / phantom (source case).

For the adaptation phase tests, the 3D lung contours of these 24 phantoms were considered and extracted. In fact, there are 9 distinct 3D lung contours reported on Figure 2. For example, a phantom with a 90B thorax and one with a 90C have the same 3D lung contours since breast and lung volumes and contours are not correlated at all. In addition, three 3DLC corresponding to other heights were created by the same process: $T_{1}, T_{2}$ and $T_{3}$ whose heights were reported on Figure 2.

\subsection{EquiVox retrieval phase performance}

In order to evaluate the performance of the EquiVox retrieval phase, the measurements of 80 different female subjects randomly selected from the CAESAR database [14] (Civilian American and European Surface Anthropometry Resource database) were considered as target cases descriptions. The latter is a database of over 2000 optical scans of Italian and Danish male and female subjects. Some of their measurements (age, sex, origin, and weight) are also stored with these scans and the spatial resolution enabling calculation of chest girth, cup size, and the height of each subject.

IRSN experts determined 5 sets of subject characteristics which influence the pulmonary anthroporadiametry dose computations. The weights $\omega_{k}$ of the associated descriptors from the set influencing the phantom choice the most were set at 4 , whereas the weights of those with no influence on that type of computation were set at 0 . In the case of in vivo counting, it is known that the chest circumference and lung volumes are the most important parameters [15]. Hence, their associated weights were given the highest value: 4 . Moreover, in this example, the weights associated to the internal volumes were set at 0 .

For each target case, we compared the source case the expert would have chosen to the classification proposed by the EquiVox retrieval phase. For 75 target cases, the experts and the EquiVox retrieval phase chose the same source case first. Thus, 5 times, the EquiVox retrieval phase put the source case chosen by the experts in second place. Consequently, in $93.75 \%$ of the cases, EquiVox chose the most accurate source case regarding the target case description. The 5 target cases, for which the EquiVox retrieval phase missed the most accurate solution, can be explained by the influence of all other informed descriptors (age, height, weight, etc.). In fact, the difference between the values of these descriptors in these 5 target cases adds up and leads to a low similarity index.

In addition, when no descriptor weighting was assigned $\left(\omega_{k}=1 \forall k \in\right.$ $\{1, \ldots, 14\})$, the EquiVox retrieval phase put the most accurate source case in first place only 54 times. 


\subsection{Performance adapations of lung contours}

As explained in the previous part of this paper, we tested two main configurations for EquiVox adaptation (one considering the phantom' heights inferior to the target' one and one considering the phantom' heights superior to the target'one) and four possibilities for each configuration.

Table 3 shows the results obtained with the first configuration (when $\Delta_{h}>0$ ). For the interpolation of $T_{1}$, the best results were obtained when $P_{3}$ was in the validation set and the worst with $P_{6}$ in it instead. For $T_{2}$, the most accurate adaptation was obtained when $P_{7}$ was in the validation set and the least one with $P_{6}$. Concerning $T_{3}$, including $P_{7}$ in the validation set gave the best interpolations whereas including $P_{3}$ gave the worst.

Table 3. Distances between interpolated and expected points with the first configuration $\left(\Delta_{h}>0\right)$.

\begin{tabular}{cccccc}
\hline \multirow{2}{*}{3 DLC } & & \multicolumn{3}{l}{ Possibility (phantom of the validation set) } \\
\cline { 3 - 6 } & $\begin{array}{c}\text { Deviation } \\
{[\mathrm{mm}]}\end{array}$ & $\# 1\left(P_{2}\right)$ & $\# 2\left(P_{3}\right)$ & $\# 3\left(P_{6}\right)$ & $\# 4\left(P_{7}\right)$ \\
\hline$T_{1}$ & Mean & 1.8 & $\mathbf{1 . 2}$ & 3.1 & 1.5 \\
& Standard & 0.7 & $\mathbf{0 . 8}$ & 1.2 & 0.5 \\
\hline$T_{2}$ & Mean & 2.1 & 1.5 & 3.4 & $\mathbf{1 . 3}$ \\
& Standard & 0.8 & 0.8 & 1.2 & $\mathbf{0 . 4}$ \\
\hline$T_{3}$ & Mean & 0.9 & 2.5 & 1.7 & $\mathbf{0 . 5}$ \\
& Standard & 0.3 & 1.2 & 0.6 & $\mathbf{0 . 2}$ \\
\hline
\end{tabular}

Generally, we can remark that $P_{6}$ always provided the highest errors and none always gave the best interpolation accuracy. Finally, we can note very important differences between best and worst deviations: the best interpolations were more than twice more accurate than the worst ones.

Usually, experts use phantoms described with $1.8 \mathrm{~mm}$ by $1.8 \mathrm{~mm}$ by $4.8 \mathrm{~mm}$ voxels. Regarding this constraint, the best adaptations were satisfying whereas the worst could infer some errors at the dosimetric calculations.

Table 4. Distances between interpolated and expected points with the second configuration $\left(\Delta_{h}<0\right)$.

\begin{tabular}{cccccc}
\hline \multirow{2}{*}{3 DLC } & & \multicolumn{4}{l}{ Possibility (phantom of the validation set) } \\
\cline { 3 - 6 } & $\begin{array}{c}\text { Deviation } \\
{[\mathrm{mm}]}\end{array}$ & $\# 1\left(P_{2}\right)$ & $\# 2\left(P_{3}\right)$ & $\# 3\left(P_{6}\right)$ & $\# 4\left(P_{7}\right)$ \\
\hline$T_{1}$ & Mean & 3.4 & $\mathbf{1 . 9}$ & 8.4 & 3.2 \\
& Standard & 1.7 & $\mathbf{0 . 7}$ & 2.6 & 1.1 \\
\hline$T_{2}$ & Mean & 2.4 & $\mathbf{1 . 7}$ & 5.4 & 1.8 \\
& Standard & 1.0 & $\mathbf{0 . 6}$ & 1.7 & 0.7 \\
\hline
\end{tabular}




\begin{tabular}{lccccc}
\hline$T_{3}$ & Mean & 1.2 & $\mathbf{0 . 8}$ & 0.9 & 0.9 \\
& Standard & 0.7 & $\mathbf{0 . 2}$ & 0.3 & 0.3 \\
\hline
\end{tabular}

Table 4 shows that the best results with the second configuration (when $\Delta_{h}<0$ ) were obtained with the same learning set and validation set for all the tested 3DLC: $\left\{P_{1}, P_{2}, P_{4}, P_{5}, P_{6}, P_{7}, P_{8}, P_{9}\right\}$ as learning set and $\left\{P_{3}\right\}$ as validation set. Nevertheless, the worst results were interpolated with Possibility \#3 for $T_{1}$ and $T_{2}$, and Possibility \# 1 for $T_{3}$. Furthermore, the best interpolation computed for $T_{1}$ was less satisfying than the others since the mean error is superior to the voxel dimensions commonly used by experts of radiation protection.

Higher differences can be observed between best and worst interpolations of $T_{1}$ and $T_{2}$ with this configuration than with the first one: the best interpolations were respectively four and three times more accurate than the worst ones.

On the contrary, the difference between best and worst interpolations of $T_{3}$ and this configuration were less important than the one with the other. A partial explanation is the distance variations of $T_{1}, T_{2}$ and $T_{3}$ from the adapted 3DLC: when $\Delta_{h}>0, T_{3}$ $(185 \mathrm{~cm})$ was adapted from $P_{8}(183.03 \mathrm{~cm})\left(\Delta_{h}=1.97 \mathrm{~cm}\right)$, whereas for $\Delta_{h}<0, T_{3}$ was adapted from $P_{9}(185.25 \mathrm{~cm})\left(\Delta_{h}=-0.25 \mathrm{~cm}\right)$. On the contrary, $T_{1}(165 \mathrm{~cm})$ was adapted from $P_{1}(164.5 \mathrm{~cm})$ when $\Delta_{h}>0\left(\Delta_{h}=0.5 \mathrm{~cm}\right)$, and from $P_{2}(167.54 \mathrm{~cm})$ when $\Delta_{h}<0\left(\Delta_{h}=-2.54 \mathrm{~cm}\right)$; and similarly, $T_{2}(179 \mathrm{~cm})$ was interpolated from $P_{6}(178.31)$ $\left(\Delta_{h}=0.69 \mathrm{~cm}\right)$ or $P_{7}(180.71)\left(\Delta_{h}=-1.71 \mathrm{~cm}\right)$.

We can notice that the best interpolations were usually obtained when using the phantom whose height is the closest.

As a remark, the adaptations performed with $\left(\Delta_{h}>0\right)$ as additional constraint were generally more accurate than the ones performed with $\left(\Delta_{h}<0\right)$. In addition, whatever the tested configuration was, the learning and validation sets had a great impact on the interpolation accuracies and important differences can be observed. Finally, we can notice that interpolations of $T_{3}$ were always twice better than interpolations of the other 3DLC.

\section{Discussion}

Figure 4 shows some interpolated lungs and their accuracies. Figure 4 a presents the most accurate lungs interpolated $\left(T_{3}\right.$ with the validation set \#4 and $\left.\Delta_{h}>0\right)$ and Figure $4 \mathrm{~b}$ the worst one $\left(T_{1}\right.$ with the validation set \#3 and $\left.\Delta_{h}<0\right)$. Each point is colored according to its interpolation error, from blue (the lowest) to red (the highest). 


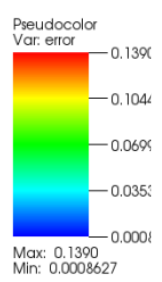

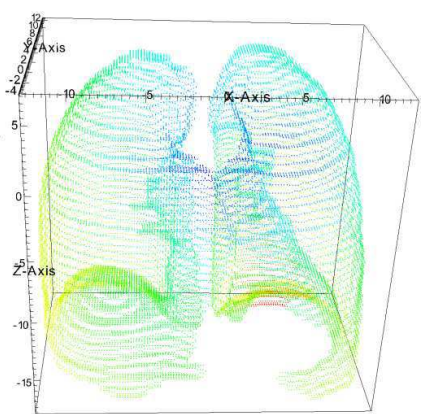

(a)

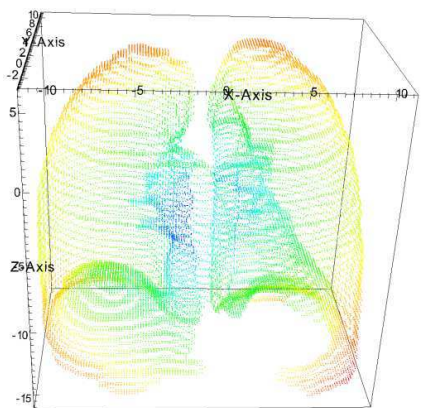

(b)

Fig. 4. Representation of an interpolated lung with: (a) $T_{3}$ with the validation set \#4 and $\Delta_{h}>0$, and (b) $T_{1}$ with the validation set \#3 and $\Delta_{h}<0$.

Since the interpolation deviations were inferior to the commonly used voxels dimensions of radiation protection experts, the best interpolations for each 3DLC were suitable. Actually, as it is visible in Figure 4, the best results we obtained allow interpolating lung contours with a suitable precision for radiation protection reports. Nevertheless, the interpolation accuracy should be increased for other domains like radiotherapy, where physicians and medicine experts also use such models as a basis for dosimetric reports.

Therefore, it emphasizes the importance of the configuration and the 3DLC chosen for each set, since the inclusion of one 3DLC in the validation set can generate an accuracy twice higher or more than another. In addition, including one 3DLC in the validation set introduced a bias for some interpolations and, at the same time, improved the accuracy of another target case (it was the case for $P_{3}$ with $T_{1}$ and $T_{3}$ when $\Delta_{h}$ was positive for example).

Indeed, EquiVox case-base is relatively young and limited. Thus, its adaptation phase is limited by the number of known 3DLC. The results presented in this study show that some 3DLC can introduce bias in the adaptation tool. These results confirm and quantify the general drawback of using interpolation as means of adaptation in CBR systems [16]: imperfections are introduced in adapted solutions.

Consequently, two ways of improvement are now considered for Equivox.

The first one consists in capitalising phantoms and 3DLC and so to ease progressively the imperfections of the solutions; the ANN interpolations, based on learning sets more and more important, will become better and better.

Nevertheless, a second option can be explored, which depends on the association of vectors to the learning set, to optimise interpolation accuracies and to determine, a priori, the best learning set/validation set for each target case. 


\section{Conclusion}

The EquiVox platform was developed for emergency situations, when a fast and reliable decision is required in order to choose the best $3 \mathrm{D}$ phantom to perform dosimetry calculation and establish a dosimetric report.

The choice is made using the CBR approach based on the feedback from previous similar experiences. EquiVox helps the experts in choosing the most similar 3D phantom by means of the computation of indices for similarity and confidence. The similarity index defines the equivalence between the target case and the source case, whereas the confidence index highlights the uncertainty in the similarity calculation.

The tests performed on an average set of target cases gave an efficiency of $93.75 \%$ in the application case of in vivo female counting for pulmonary anthroporadiametry.

Furthermore, an adaptation strategy for 3D Lung Contours (3DLC) was implemented and discussed. This strategy was based on Artificial Neural Networks. Different configurations based on different sets of 3DLC for learning and validation were tested and analysed through the interpolations of three new lung contours.

The results show the importance of the choice of the 3DLC repartition between the learning and validation sets: whereas the best interpolations met the requirements of experts, it was not always the case for the worst ones.

Some of the interpolation errors were related to the imperfections that can be contained in the source case solutions. Thus, further work will focus on the elaboration of an adaptation algorithm capable of taking into account the confidence that can be associated to a source case solution.

In other words, our goal is to propose a tool that creates rules for the adaptation of target cases using this confidence indice. Moreover, we will also extend the EquiVox adaptation to other organ contours of thorax.

\section{Acknowledgements}

The authors want to thank INSERM (Institut National de la Santé et de la Recherche Médicale), LCC (Ligue Contre le Cancer), Pays de Montbéliard Agglomération and SFRP (Société Française de Radioprotection) for their financial helps.

\section{References}

1. Broggio, D., Zhang, B., de Carlan, L., Desbrée, A., Lamart, S., le Guen, B., Bailloeuil, C., Franck, D.: Analytical and Monte Carlo assessment of activity and local dose after a wound contamination by activation products. Health Phys., vol. 96, pp. 155-163 (2009)

2. Huet, C., Lemosquet, A., Clairand, I., Rioual, J. B., Franck, D., de Carlan, L., Aubineau-Lanièce, I., Bottollier-Depois, J. F.: SESAME: a software tool for the 
numerical dosimetric reconstruction of radiological accidents involving external sources and its application to the accident in Chile in December 2005. Health Phys., Vol. 96, pp. 76-83 (2009)

3. Kolodner, J.: Case-Based Reasoning. Morgan Kaufmann Publishers (1993)

4. McCulloch, W., Pitts, W.: A logical calculus of ideas immanent in nervous activity. Bulletin of Mathematical Biophysics. Vol. 5, pp. 115-133 (1943)

5. ICRP89. Basic anatomical and physiological data for use in radiological protection. International Commission on Radiological Protection Publication 89 (2002)

6. Farah, J., Broggio, D., Franck, D.: Examples of Mech and NURBS phantoms to study the morphology effect over in vivo lung counting. Radiation Protection and Dosimetry Special Issue, Vol. 144, pp. 344-348 (2011)

7. McNeel. Rhinoceros Modeling tools for designers. http://www.rhino3d.com. [Online]

8. Christensen, G. E. Deformable shape models for anatomy. Washington University. PhD Thesis (1994)

9. Clairand, I., Bouchet, L. G., Ricard, M., Durigon, M., Di Paola, M., Aubert, B.: Improvment of internal dose calculations using mathematical models of different adult heights. Phys. Med. Biol., Vol. 45, pp. 2771-2785 (2000)

10. Hsieh, W.: Learning Methods in the Environmental Sciences - Neural Networks and Kernels. Cambridge University Press (2009)

11. Ponce, J., Brette, R.: Polynomial interpolation. Introduction to scientific computing and its applications. http://audition.ens.fr/brette/calculscientifique/2006-2007/lecture2.pdf [Online] (2010)

12. Digiteo. Scilab Home Page. http://www.scilab.org. [Online]

13. (CEN), European Committee for Standardization: Size designation of clothes: part 1. Terms definitions and body measurement procedure. EN 13402-1 (ISO 3635: 1981 modified) (2001)

14. Robinette, K M.: CAESAR. measures up, Ergonomics in Design 8(3) pp. 17-23 (2000)

15. Kramer, G. H., Burns, L. C.: Evaluation of the effect of chest wall thickness, tissue composition and photon energy on the quantity muscle equivalent chestwall-thickness by Monte Carlo simulation. Radiat. Prot. Dosim., Vol. 82, pp. 115-124 (1999)

16. Chatterjee, $\mathrm{N}$ and Campbell, J. A.: Interpolation as a means of fast adaptation in case-based problem solving. Kaiserslautern, Germany: Ralph Bergmann and Wolfgang Wilke editors, 1st German Workshop on Case-Based Reasonning, pp. 65-74 (1997) 